\title{
Quaderni
}

QUADERN I Communication, technologies, pouvoir

73 | Automne 2010

La métropole parisienne entre récits, paroles et échanges

\section{Paris-Métropole à l'épreuve du vécu métropolitain des quartiers gentrifiés du nord-est de Paris}

\section{Sophie Corbillé}

\section{(2) OpenEdition}

Journals

Édition électronique

URL : http://journals.openedition.org/quaderni/451

DOI : 10.4000/quaderni.451

ISSN : 2105-2956

Éditeur

Les éditions de la Maison des sciences de l'Homme

Édition imprimée

Date de publication : 5 octobre 2010

Pagination : 75-88

Référence électronique

Sophie Corbillé, "Paris-Métropole à l'épreuve du vécu métropolitain des quartiers gentrifiés du nordest de Paris », Quaderni [En ligne], 73 | Automne 2010, mis en ligne le 05 octobre 2012, consulté le 19 avril 2019. URL : http://journals.openedition.org/quaderni/451 ; DOI : 10.4000/quaderni.451 


\section{$D$ ossier}

\section{Paris-Métropole}

à l'épreuve du vécu

métropolitain des quartiers gentrifiés

du nord-est de Paris

L'histoire des relations matérielles et symboliques entre Paris et les communes alentours oscille entre clôture et ouverture, domination et coopération, mépris et désir. Sans compter le poids des représentations qui obscurcissent parfois la compréhension des échanges, menant souvent à privilégier la dichotomie capitale/ banlieue ${ }^{1}$. Depuis 2001, avec la création d'un poste d'adjoint au maire chargé de la coopération avec les collectivités territoriales d'Île-de-France, la ville de Paris affiche clairement sa volonté d'établir des relations de coopération dans des domaines tels que le logement, les transports ou la culture. À cette initiative, et dans le cadre du projet Paris-Métropole, s'ajoute la volonté de créer une communauté d'intérêts partagés, de «faire émerger une culture commune de l'agglomération parisienne », et de faire de ce territoire un «espace vécu » partagé, s'appuyant sur une série d'événements, d'espaces de débats et d'opérations de communication visant le grand public et les citadins de la métropole (conférences, séminaires, publications en ligne, etc. $)^{2}$.

\section{Sophie Corbillé}

Maître de Conférences Université de Paris Sorbonne CELSA
La politique actuelle de métropolisation s'inscrit en réalité dans une histoire urbaine longue marquée par de nombreux débats sur la «bonne» taille de l'agglomération parisienne et la place de Paris, en tant que ville et capitale, dans cet ensemble. À l'heure de la globalisation, elle apparaît aussi comme une réponse pour faire face aux processus de fragmentation qui caractérisent la production de l'urbain et de la ville aujourd'hui. Faire métropole, c'est en effet l'espoir de "faire lien » afin de lutter contre une société urbaine désormais éclatée entre espaces gentrifiés, relégués et périurbains (Donzelot, 2004) et partagée entre d'un côté des villes en réseau interconnectées et de l'autre 
des mégapoles tentaculaires (Mongin, 2004).

À partir d'une enquête ethnographique menée dans les quartiers gentrifiés du nord-est de Paris ${ }^{3}$, cet article a pour objectif d'interroger le vécu métropolitain des nouvelles classes moyennes et supérieures venues vivre dans ces arrondissements $\left(\mathrm{X}^{\mathrm{e}}, \mathrm{XI}^{\mathrm{e}}, \mathrm{XII}^{\mathrm{e}}, \mathrm{XVIII}^{\mathrm{e}}, \mathrm{XIX}^{\mathrm{e}}, \mathrm{XX}^{\mathrm{e}}\right) . \mathrm{Si}$ les processus de gentrification à l'œuvre à Paris depuis les années 1970, encouragés par les politiques urbaines visant à rééquilibrer Paris et la région, tendent à atténuer les disparités socioéconomiques qui ont longtemps prévalu entre les arrondissements populaires de l'Est et les quartiers bourgeois de l'Ouest ${ }^{4}$, c'est en réalité davantage à une redéfinition de l'opposition Est/Ouest qu'à sa disparition qu'on assiste : l'installation des nouvelles classes moyennes et supérieures autour des rues de faubourgs et des anciens villages du nord-est de Paris a en effet favorisé l'émergence d'un monde urbain, d'un nouvel espace symbolique et matériel avec lequel ces nouveaux venus entretiennent une relation de connivence 5 . Sur quelles logiques ce monde urbain du nord-est de Paris repose-t-il ? Quelles sont les frontières de ce monde ? Et dans quelle mesure les processus de gentrification ont-ils ou non réorganisé la relation entre Paris et les banlieues limitrophes?

Cet article propose d'explorer deux hypothèses. Le vécu métropolitain de ces acteurs repose en premier lieu sur une forme de vie urbaine dans laquelle on trouve à la fois l'expression d'un mode de vie, l'instauration d'un ordre social urbain et l'attachement fort au « local», mais aussi le fait de vivre une expérience, entendue ici au regard de trois éléments. D'abord, la place donnée à « l'am- biance » des espaces. Ensuite la place de l'individu pour comprendre le lien entre ces personnes et les lieux, leur relation à l'espace ne se limitant pas à l'identification sociale ni à la constitution d'un territoire. Enfin, le caractère hétérogène de la relation des acteurs à la ville ${ }^{6}$. La seconde hypothèse concerne les frontières de ce vécu métropolitain : dans la mesure où vivre dans ce monde urbain, c'est aussi affirmer pleinement son identité de Parisien au sens de faire l'expérience à la fois d'une ville et de la grande ville, leur métropole s'arrête souvent aux frontières de Paris.

\section{Parcours résidentiels dans le nord-est de Paris}

À la fin des années 90, Benjamin et Anne décident de quitter Abbesses (XVIII ${ }^{\mathrm{e}}$ arr.) suite à la naissance de leur fille. Benjamin, médecin de formation et exerçant dans l'édition médicale, appartient à la « bourgeoisie internationale newyorkaise ». Anne, de son côté, travaille dans le milieu du cinéma et a été élevée dans la grande bourgeoisie parisienne. La quarantaine, disposant d'un capital à un moment où les prix du marché commencent à augmenter, ils choisissent de devenir propriétaires. Leur recherche porte sur quatre arrondissements de Paris, les $\mathrm{X}^{\mathrm{e}}, \mathrm{XI}^{\mathrm{e}}, \mathrm{XIX}^{\mathrm{e}}$ et $\mathrm{XX}^{\mathrm{e}}$. Ils traversent aussi le périphérique pour chercher en banlieue proche, à Bagnolet, Montreuil et au Pré-Saint-Gervais, mais abandonnent rapidement l'idée : «En fait, à chaque fois qu'on tombait sur des trucs qui étaient vraiment intéressants (...), on s'apercevait qu'il n'y avait pas de café. Et moi, pour avoir toujours vécu à Paris, j'adore les cafés, j'adore les bistrots, j'adore la vie de quartier ». Début 2000, Anne et Benjamin achètent un loft dans une ancienne imprimerie 
réhabilitée par un marchand de biens au nord du $\mathrm{X}^{\mathrm{e}}$ arrondissement. Belleville répond à leurs critères: «Et donc Belleville parce qu'outre le fait que c'était un quartier accessible en termes d'achat, ça faisait aussi partie des quartiers qu'on aimait bien pour plusieurs raisons. Le fait que c'était populaire et mélangé, le fait qu'il y ait aussi bien des Juifs, des Chinois, des Arabes, des Turcs que des Africains, etc. Moi c'est ça quej'adore (...)».

Éric, jeune designer, vit avec son amie dans un ancien atelier d'un passage du $11^{\mathrm{e}}$ arrondissement qu'il a transformé en " espace ouvert». À ses yeux, l'intérêt de ce quartier à l'ombre du Faubourg Saint-Antoine réside dans la qualité des relations sociales : «ici, c'est se retrouver dans un endroit un peu différent dans Paris, dans un lieu un peu plus intime, plus vie de quartier, petit village tout en étant dans Paris. Moi, je trouve ça génial. Parce que tout de suite tu penses à la convivialité, ce que permettent aussi les petites ruelles sympas avec de la verdure, où il n'y a pas de passage de bagnoles, etc. (...). » Auparavant, il avait vécu dans une maison à Montreuil. «Et c'est vrai que c'est toujours plus vers l'Est $q u$ 'on est partis. On s'est retrouvés vers Ménil, Belleville, le canal Saint-Martin, même Gambetta et on a passé le périph et on s'est retrouvés à Montreuil et ça c'est récent Montreuil, sept ans environ. Maintenant Montreuil ça commence un peu à saturer. Tu appelles la mairie pour avoir des lieux, à l'époque tu avais plein de trucs, maintenant ça devient vraiment dur. Donc on va aller peut-être vers Bagnolet, les Lilas, le Pré Saint-Gervais. Mais Montreuil ça a été vraiment le XXIe arrondissement $»$.

Gilles, la quarantaine, issu d'une famille bour- geoise, travaillant chez France Télécom, peintre à ses heures perdues et résidant au moment de l'entretien dans un logement social du $\mathrm{XX}^{\mathrm{e}}$ arrondissement, est certainement celui qui exprime avec le plus de force son rapport exclusif à ce monde urbain, un espace pour lui contenu dans les frontières de Paris intra muros : «Je n'ai jamais vécu dans l'ouest de Paris. Moi, je suis vraiment de l'est. J'ai vécu à Bastille, Ménil, Gambetta et maintenant dans le $X X^{e}$, près de la place de la Réunion. J'adore ces endroits. Par exemple, quand tu prends la rue du Faubourg-du-Temple ou le boulevard de Belleville, c'est dingue, tu as de tout, des Chinois, des Blacks, plein de gens différents, de l'animation. Moi, je suis vraiment dedans, de l'est. Je ne me sens pas bien ailleurs ».

\section{Le village urbain, un espace " plié »}

Dans les raisons données de vivre dans les quartiers du nord-est de Paris, le critère économique est souvent mentionné en premier. D'autres éléments, considérés objectifs, apparaissent aussi : la situation centrale du quartier, les transports publics et la présence d'espaces verts. La question scolaire peut également influencer leur choix même si les ressources dont ces acteurs disposent pour s'affranchir de la carte scolaire rendent ce critère moins contraignant. Mais ces raisons ne suffisent pas à elles seules à expliquer leur choix. À les écouter, ces quartiers offriraient aussi une forme de vie urbaine particulière.

Celle-ci se caractérise d'abord par sa physionomie. Dans les entretiens, les personnes évoquent les petites rues et les passages qu'elles opposent aux boulevards haussmanniens des quartiers bourgeois ; les immeubles bas et les anciens ate- 
liers qui côtoient des immeubles plus modernes ; enfin l'aspect vallonné des quartiers. À cela est associée une sociabilité particulière. Ces espaces seraient favorables à l'épanouissement d'une vie sociale circonscrite à un petit territoire, cadre privilégié d'une sociabilité marquée par l'interconnaissance et la proximité : ici, on connaîtrait ses voisins mais aussi les commerçants. Pour finir, les personnes ne manquent pas de mettre en scène la cohabitation des âges, des origines sociales et des cultures. On retrouve ici le «quartier-village» décrit par S. Chalvon-Demersay, ce «cadre rêvé d'une convivialité idéale » ${ }^{7}$.

Mais le quartier-village n'est pas seulement un décor ni une illusion, il est aussi le résultat de pratiques marquées par l'envie de faire des choses ensemble : ces habitants ne cessent en effet d'instaurer des situations d'échange qui leur permettent de se rassembler et de se lier au quartier, établissant des réseaux ou des petits groupes de nature et de force diverses. Les observations menées montrent que ces habitants font relation selon trois modalités : celle de l'acteur urbain, de l'ami et de l'habitant de quartier. Agir en acteur urbain c'est considérer que le lieu de résidence n'est pas simplement un cadre de vie dont on aurait des usages, mais qu'il doit être également l'objet d'attentions, de discussions et d'engagements, attitudes d'ailleurs encouragées par les pouvoirs publics. Le conseil de quartier et les associations de quartier sont les instances principales de cette manière $\mathrm{d}$ 'agir, un agir parfois surtout communicationnel. Autre façon de faire des choses ensemble, se réunir entre amis. L'importance des réseaux d'amis dans la relation au quartier est évidente : c'est parfois par un ami que les personnes entrent dans le quartier, elles font aussi venir des proches quand un appartement se libère et elles deviennent parfois amies dans le cadre résidentiel ou scolaire. Le lien amical, qui repose sur une relation d'obligation réciproque, participe fortement à la construction du quartiervillage et dans bien des cas le voisin n'est pas cet étranger familier avec lequel on entretient une relation de réserve ${ }^{8}$, mais un ami avec lequel on échange ses difficultés et ses joies, avec lequel on s'entraide matériellement. Dernière manière de faire lien, se comporter en habitant de quartier. Cela implique, au-delà des processus de ségrégation et de distinction à l'œuvre dans les espaces résidentiel et scolaire, de reconnaître l'autre comme partageant le même espace en ville, attitude qui repose sur des interactions fonctionnant sur le mode de la familiarité et de la convivialité. Cette identité citadine spécifique émerge à l'occasion d'événements circonscrits qui forcent les uns et les autres à se rencontrer par exemple lors d'un vide-grenier.

Le quartier-village de ces acteurs n'est donc pas un espace plan et homogène, mais il est un espace «plié »" ${ }^{9}$. Il repose sur des pratiques et des représentations diverses, obéissant à des logiques a priori contradictoires mais complémentaires. D'un côté la constitution d'un entre-soi parfois exclusif à l'occasion de relations amicales se déroulant dans les espaces résidentiels ou dans des lieux de sociabilité tels que les bars ou associations ; de l'autre des relations plus éphémères mais essentielles avec d'autres habitants du quartier, dans des lieux circonscrits et à l'occasion d'événements ou de relations d'approvisionnement. La discontinuité apparaît donc comme un élément clé de ce mode de vie, leur quartier étant constitué de toutes ces situations d'échanges. 
Si le quartier-village se caractérise par la mise en œuvre d'un mode de vie convivial, il est cependant un lieu fragile, soumis à des déséquilibres. Il y a d'abord le regret de la « boboisation », expression qui désigne la fragilisation de l'équilibre social, rappelant les comportements des habitants du $14^{\mathrm{e}}$ arrondissement au début des années 80 à la recherche à la fois de l'entre-soi et de la diversité (Chalvon-Demersay, 1984, p.154). Mais une autre notion est aussi centrale chez ces acteurs, celle d' « oberkampfisation ».

\section{L'oberkampfisation, de l'ordre social à la perte du « local »}

Vanessa a ouvert début 2000 un restaurant rue Oberkampf, devenu depuis un des endroits à la mode de la rue. Aujourd'hui, elle se dit déçue par le quartier : « je n'aime pas la rue Oberkampf. Peut-être parce que je suis trop allée dans le haut de la rue quand on travaillait à La Favela. Il y avait vraiment un esprit village encore et moi j'étais vraiment bien dans ce quartier. Mais là, quand j'y vais maintenant, c'est vraiment la faune, il y a trop de racailles. Oui ça devient un peu comme Bastille et pour moi c'est un peu Disneyland. Ça n'a aucun intérêt ». Marie, dont la boutique-brocante est à deux pas, fait le même constat : "en deux ans j'ai vu beaucoup de changements dans le quartier, malheureusement je dirais que c'est dans le sens négatif. J'aurais préféré que des commerçants s'installent audessus, à la place d'avoir des paninis comme à Saint-Michel. (...) En bas, là, c'est bien, c'est la plus belle partie parce que tu as des vrais commerçants (...). Alors qu'en haut tu n'as pas ça. Et avec ces trucs de paninis, tu as l'impression d'être à Saint-Michel, ça te ramène tous les petits z'y-vas du coin et c'est ce qui s'est passé à Bastille il y a quelques années ».

Venues dans ce quartier qui offrait à leurs yeux une sociabilité de village, elles expriment une forme de désenchantement. Les commerces dits « de quartier » auraient disparu au profit de commerces de divertissement et « bas de gamme », attirant d'autres populations : la « racaille » et les «z'y-vas du coin », les «fils à papa» des beaux quartiers et depuis peu les touristes. Dans le récit qu'elles font, c'est l'équilibre entre les gens du quartier et ceux extérieurs qui est en jeu, entre ceux d'ici et ceux d'ailleurs, équilibre qui résulte d'un travail de définition de ce qu'est le « local », activité qui dessine en creux un ordre social du lieu. Quand les acteurs parlent de ces changements, ils distinguent en effet, sans en avoir conscience, le local du résidentiel : toute personne résidant dans un quartier n'est pas nécessairement considérée comme « locale ». Cela apparaît clairement dans le discours de Vanessa qui considère que les jeunes qui fréquentent Oberkampf, pour la plupart issus de l'immigration, ne sont pas du quartier. Ce processus de labellisation concerne aussi les commerçants : le vendeur de panini ou le cafetier « chinois » peut bien habiter la rue mais il se verra plus difficilement qualifier de commerçant de quartier. À cela s'ajoute un autre processus menaçant l'identité des lieux : l'uniformatisation des paysages urbains, pouvant aller jusqu'à leur « disneylandisation ». «Alors je ne supporte plus le faubourg Saint-Antoine. Je trouve que c'est massacré, c'est de pire en pire. (...) Ça me fait un peu penser à Londres avec toutes ces chaînes de magasins qui s'installent, ou à la rue de Rennes. (...) Et la conséquence de ça, c'est que peu à peu, il n'y a plus vraiment 
d'identité au lieu. ", dit Eric. Ce qu'il regrette, c'est le remplacement de "l'ébéniste » par le «Mac Donald», c'est la perte de l'identité du lieu que le passage à une consommation standardisée et mondialisée entrainerait. Dans ce jeu de comparaisons auquel ces «images-repoussoirs » donnent lieu (Ibid., p.144), c'est la particularité du lieu qui est en jeu, l'homogénéisation des paysages affaiblissant la différence entre le « $i c i$ » et le «ailleurs ». Le processus d'«oberkampfisation» se produit donc quand un air de déjà-vu s'installe.

Le quartier-village est donc le lieu du mode de vie et de l'ordre social. L'espace urbain est considéré comme le support de relations relevant de l'inter(re)connaissance permettant de " faire société ». Il est aussi l'espace de l'appartenance et de l'identité : dans la relation aux lieux, les personnes s'identifient aux autres habitants et aux autres «bobos », construits comme des semblables. Cet espace est enfin celui de processus de domination, voire de séparation, pouvant être imputés à l'inquiétude de certaines personnes qui voient leur monde urbain menacé par l'arrivée d'autres populations et leur position fragilisée par l'évolution du marché immobilier. On serait là dans une logique du territoire, où "l'autre » est cependant à géométrie variable : s'il est parfois clairement identifié et placé au pôle négatif comme étranger («les jeunes», «les Chinois»), il peut être aussi perçu positivement, étant alors davantage du côté de l'étrangeté.

\section{La grande ville : faire l'expérience enchantée de la diversité}

Ce qui constitue en effet aussi à leurs yeux le charme de ces espaces, c'est leur dimension mul- ticulturelle. Et l'émerveillement est à son comble quand les différences aboutissent à des métissages improbables, à l'instar du «Chinois casher » du boulevard de Belleville évoqué par l'un des interlocuteurs. Ces quartiers sont dès lors construits comme des sortes de territoires ethnographiques au sens où les individus sont dans un rapport de découverte à l'espace et aux autres citadins, considérés avant tout comme des groupes culturels : Arabes, Turcs, Juifs ou Chinois, ou encore " bobos ", eux-mêmes apparaissant comme un groupe parmi d'autres (Corbillé, 2009).

La relation enchantée à "l'autre » se déroule essentiellement dans l'espace public et marchand, ces lieux constitutifs de «l'ambiance » du quartier, une ambiance régie par des interactions caractérisées par leur plasticité : les échanges sont souvent brefs, anonymes et possèdent une dimension sensorielle importante. Il faut également souligner la place importante qu'occupent les espaces marchands du type de ceux décrits par E. Lallement à Barbès, des lieux ouverts sur l'espace urbain qui offrent la possibilité de mettre en scène une société multiculturelle pacifiée (2009). Il n'est pas étonnant alors que la rue du Faubourg-du-Temple ou celle du FaubourgSaint-Denis constituent des pôles d'attractivité : c'est ici que les acteurs font l'expérience du jeu identitaire en exprimant à la fois une attention accrue aux différences mais aussi une certaine indifférence à leur encontre, oscillant entre distance et proximité. La diversité enchantée ne serait-elle pas alors l'un des éléments-clés de la grande ville contemporaine, offrant une manière particulière de jouir de l'anonymat et des différences ? Et ne serait-elle pas aussi une façon de faire l'expérience de la globalisation, d'éprouver 
l'accélération de la circulation des êtres humains, des marchandises, des capitaux et des images.

Avec la grande ville cosmopolite, monde où l'anonymat et l'étranger prévalent, organisé autour du mouvement et du déplacement, l'espace urbain est considéré comme le lieu d'une expérience au sens où les individus éprouvent la ville dans sa surface au moyen de la perception, faisant d'elle un objet esthétique à regarder et dont ils attendent, inversement, qu'elle produise un effet sur eux : être émerveillés ou bousculés sont autant d'expériences recherchées et que ces quartiers offriraient, à l'inverse des arrondissements de l'ouest décrits comme « monotones», « ennuyeux ». Le souci de l'animation amène d'ailleurs certains interlocuteurs à considérer qu'une pointe de danger agrémente l'attrait de l'espace. La grande ville est donc celle de la rue, notamment de la rue de faubourg commerçante et dense, une ville considérée à l'aune de l'ambiance et de l'effet qu'elle produit, une ville qui offre une « esthétique de la distraction » (Benjamin, 1991). Mais c'est aussi celle de l'individu, qui, en raison de l'excitation proposée par les situations urbaines, fait l'expérience de brefs déplacements qui le forcent à se «désajuster» et à faire preuve de petites réflexivités au quotidien.

\section{« Ici, c'est branché », la quête de la différence et du singulier}

Si les interlocuteurs apprécient les commerces authentiques, ils parlent tout autant des cafés à la mode. En fait, les discussions autour des « lieux branchés » constituent un leitmotiv, l'occasion d'évoquer le nouveau restaurant à fréquenter ou la dernière boutique de vêtements où se rendre.
Les personnes font aussi parfois le lien entre leur parcours résidentiel et l'évolution des lieux à la mode. Gilles, en retraçant ses différents lieux de résidence remarque qu'il a toujours un peu devancé ou suivi la mode ; et Anne et Benjamin expliquent qu'ils ont fui Abbesses devenu «super branché ». Les discours sur la mode peuvent prêter à sourire. Ils peuvent aussi agacer : on est dans le domaine du futile, de l'apparence. Reste que ce phénomène n'est pas sans effet sur ces quartiers et il suffit d'observer les berges du canal SaintMartin pour s'en convaincre. Espace peu occupé au début des années 90, il attire aujourd'hui une foule de personnes qui s'y pressent dès que les beaux jours arrivent.

Que serait un "lieu branché »? Il est d'abord souvent associé à une histoire : un espace dégradé, à la marge est découvert par quelques « pionniers » qui l'investissent, proposant quelque chose de nouveau qui rencontre un succès, timide puis important. C'est ce que raconte Éric : «Bastille, il y a 15 ans, c'est devenu le lieu à la mode, branché. Au départ c'était un endroit où il y avait plein de choses à faire, un lieu où tu te retrouvais avec tout à construire même s'il y avait des choses existantes, mais c'était en fin de course. Une vague de créateurs a eu lieu, ils sont venus avec toutes leurs idées. Et puis, au moment où ils avaient tout puisé, ils se sont délocalisés et ils sont allés vers Oberkampf. ». Le lieu branché est donc associé au processus de gentrification et son histoire croise le récit de la frontière et de la conquête. Deuxième élément, l'importance de la consommation. Presque toujours, le lieu à la mode s'organise autour de quelques restaurants, ateliers d'artistes, cafés ou galeries. Sur ce point, les media jouent un rôle clé : de nombreux articles 
portent sur ces quartiers, indiquant les dernières « tendances », là « où boire un verre » et là « où sortir», offrant une nouvelle image des espaces, présentés comme des centralités ludiques. Le «lieu branché » ce serait donc la rencontre d'un espace, d'un projet et d'un discours médiatique. Enfin, dernières caractéristiques de ces espaces : la nouveauté et la différence, deux qualités dans lesquelles se logerait l'effet du lieu «branché », celui d'attirer. À cet égard, le travail de construction du « local » décrit précédemment participe pleinement au phénomène de la mode.

Un «lieu branché » a aussi de particulier son statut incertain : il oscille entre le lieu peu connu et celui déjà très connu; entre le lieu unique mais semblable à d'autres. On retrouve ici la spécificité du phénomène de la mode, cette «forme de vie » tendue entre «le besoin de saisir le singulier » et «l'aspiration au général » (Simmel, 1988, p.88). Le «lieu branché » fonctionne dès lors un peu comme un «lieu exemplaire » (Micoud, 1991) : un lieu singulier qui illustre, un spécimen au sens d'un cas singulier dans une série, digne dès lors d'être imité, de faire modèle. On comprend mieux pourquoi ces lieux, tout en étant singuliers, entretiennent toujours un air de famille avec les autres de la série. Ce statut incertain explique certainement le rapport ambivalent des personnes à ces lieux : ils attirent autant qu'ils déçoivent. D'un côté, c'est parce qu'Oberkampf possède un air de ressemblance avec Bastille que cette rue acquiert, à un moment donné, le statut de lieu branché. Mais quand la similitude est trop forte, les interlocuteurs adoptent une attitude blasée envers ces espaces qui les avaient séduits. On retrouve ici le processus d'oberkampfisation, l'effacement de la différence entre le «ici» et le « ailleurs ».
Pourquoi les acteurs sont-ils sensibles à la recherche du "lieu branché »? En racontant qu'on a découvert un restaurant où «ça vaut le coup d'aller » ou en ne fréquentant plus Bastille devenu trop «tendance », ils mettent en exergue le fait qu'ils ne font pas tout à fait pas comme les autres et qu'ils ne le sont donc pas. Se joue là « la volonté de paraître "au fait"” " et de «devenir-remarquable » (Simmel, ibid.), le «bobo » apparaissant souvent comme le représentant de cette attitude. Mais le souci de particularisation s'accompagne toujours d'une opération inverse qui tend à l'annuler, l'imitation. D'ailleurs, le «bon plan » ne doit-il pas l'être également pour d'autres.

L'expérience métropolitaine est donc indissociable des lieux branchés qui constituent ce qu'A. Bourdin appelle des "lieux générateurs de la forme urbaine » $(2005$, p. 81), nouvelles centralités qui fonctionnent sur une «dynamique compétitive » de la concurrence et de l'attractivité, menant à la logique du palmarès. La mode oblige dès lors à repenser la fabrication de l'urbain qui se fait aujourd'hui selon un double processus : la différenciation (la recherche de l'original) et l'imitation (la réplique, la copie). Pour M. Roncayolo, qui analyse l'érosion de la géographie symbolique de Paris, on passerait en réalité à « une sorte d'expérimentation. Il y a des modes, voilà tout ce qu'on peut dire. On essaie des lieux, dans des opérations souvent éphémères » (1994, p. 318). L'observation des quartiers du nord-est de Paris confirme et nuance cette conclusion. C'est dans le tissage des deux modalités décrites précédemment que réside la spécificité de l'expérience urbaine et des identités citadines : la construction du local nourrit la fabrique des 
lieux branchés, la quête du singulier accompagne l'émergence de groupes affinitaires, l'étranger est parfois « indigénisé », tandis que l'autochtone se fait étranger et les liens faibles à l'œuvre dans les « espaces d'ambiance » alimentent l'attachement aux lieux.

\section{Paris-métropole : relation d'identité versus relation d'altérité}

Les processus de gentrification semblent atténuer la dichotomie Paris/banlieue. Certaines personnes rencontrées ne disent-elles pas vouloir vivre aussi bien dans les quartiers du nord-est de Paris que dans les villes limitrophes, notamment les anciennes banlieues rouges qui connaissent elles aussi de profondes transformations socioéconomiques ? Sans compter que ces communes cherchent à attirer ces catégories de populations, non sans succès dans un contexte où les prix immobiliers ne cessent d'augmenter. Pour la presse, qui dès les années 2000 parle d'une véritable «ruée vers l'est », le processus irait bien au-delà du périphérique : «Vous avez la bougeotte et vous ne roulez pas sur l'or? Alors oubliez l'ouest parisien un peu figé, ultra-classique, et surtout hors de prix. Cap à l'Est ! Cap sur Paris XIII', la nouvelle rive gauche. Cap sur le Val-de-Marne, la Seine-et-Marne ou la Seine-Saint-Denis, où le mètre carré est de $15 \%$ à $20 \%$ moins cher que dans les Hauts-de-Seine. Le "Far East", Montreuil ou Ivry-sur-Seine, Marne-la-Vallée ou Magny-le-Hongre, la nouvelle frontière des "bobo", des stars-uppers, des plasticiens, des jeunes couples avec enfants qui fuient les particules de diesel de la capitale $\gg{ }^{10}$.

Il faut dire que les acteurs de la ville travaillent depuis plusieurs décennies à faire de cette frontière autre chose qu'une coupure. Certains aménagements urbains menés par la ville de Paris, notamment ceux réalisés aux portes de Paris dans le cadre des Grands Projets de Rénovation Urbaine, visent ainsi à fabriquer de la continuité entre les arrondissements du nord-est et les communes limitrophes. Les projets autour du périphérique, comme ceux des tours, s'inscrivent également dans une réflexion menée par les politiques, les architectes et les urbanistes sur la recomposition de cette frontière. Dernier exemple, le prolongement des lignes de métro en banlieue qui favorise le rapprochement de Paris et de la banlieue et joue, pour les acteurs rencontrés, un rôle important dans leur décision de traverser le périphérique.

Mais si de la continuité se fabrique entre Paris et les communes alentours, Paris reste néanmoins le centre de gravité de l'expérience urbaine de ces acteurs. La banlieue n'a ainsi droit d'entrée dans ce monde urbain que quand elle est finalement «confondue » ou «absorbée » dans Paris, à l'instar d'un Montreuil décrit comme le «XXI arrondissement de Paris »; ou bien quand elle est « reliée " à la capitale donnant le sentiment aux personnes d'être finalement presque à Paris. Et quand ces acteurs quittent Paris pour s'installer en banlieue, c'est bien souvent vers une autre expérience urbaine qu'ils se tournent. Outre l'opportunité souvent de devenir propriétaires, c'est le choix d'une autre vie en ville qui est fait, une ville qui offrirait une meilleure qualité de vie, surtout pour les familles : habiter un pavillon et disposer de plus d'espace, bénéficier des joies d'un jardin, être à proximité de grands espaces verts, etc. Comment comprendre la persévérance de cette 
frontière symbolique ? Cela tient notamment à un dernier élément-clé de ce vécu métropolitain, l'attachement à l'identité de Parisien. Ce qui se joue dans le fait de résider dans les quartiers du nord-est de Paris, c'est en effet aussi l'affirmation d'une appartenance à Paris en tant que ville singulière mais aussi grande ville. Cela est apparu clairement dans les entretiens. Pour ces acteurs, habiter Belleville, Ménilmontant ou Gambetta, c'est vivre dans les vrais quartiers parisiens, comme si ces espaces étaient dépositaires de l'identité et de l'histoire de la capitale, une identité qui plonge ses racines dans la ville moderne des $\mathrm{XIX}^{\mathrm{e}}$ et $\mathrm{XX}^{\mathrm{e}}$ siècles : les références aux classes populaires voire dangereuses (par exemple les Apaches), aux vagues successives d'immigration, aux ateliers d'ouvriers et d'artisans, aux luttes politiques et aux traditions militantes, à la liberté qu'offrirait ce monde urbain où il est encore possible de flâner dans ses rues commerçantes ou à une convivialité quasi «titiparisienne » des passages et des anciens villages, montrent bien qu'en habitant dans ces quartiers, c'est le choix de Paris qu'ils font. Le lien très fort qu'ils entretiennent au « local», à leur quartier-village multiculturel dans lequel ils se comportent en habitant de quartier, est donc toujours aussi l'affirmation de leur appartenance parisienne, renforçant de cette manière l'identité du Paris aux 80 villages.

Les politiques urbaines de requalification menées depuis le début des années 80 sur les quartiers du nord-est de Paris ne sont pas étrangères à ce processus : reconnaissance des spécificités socioculturelles de ces quartiers, réhabilitation du bâti résidentiel à travers des opérations programmées d'amélioration de l'habitat ou encore valorisation du patrimoine historique à l'instar de la restaura- tion de l'ancien siège de la CGT, la Maison des Métallos, devenue un centre socioculturel $\left(\mathrm{XI}^{\mathrm{e}}\right)^{11}$. Le développement d'un marketing de la consommation culturelle y participe également : les bars de la rue Oberkampf reposent sur une mise en scène des traces d'un passé ouvrier et populaire conservé, réinventé ou totalement imaginé ; cour Saint-Emilion $\left(\mathrm{XII}^{\mathrm{e}}\right)$, de grandes photos en noir et blanc évoquent l'activité viticole du lieu ; et les marchands de biens qui réaménagent les espaces industriels prennent soin de mettre en valeur «l'esthétique » attachée à ces anciens lieux de travail. Ce travail de mise en forme de ce « vieux Paris populaire », qui s'accompagne d'une marchandisation des identités, participe clairement à l'attrait exercé par ces lieux sur ces acteurs, même s'il porte aussi en lui les germes du désenchantement des lieux. L'identité de ce Paris-là repose largement sur la «parole mythique » qui favorise la forme sur le sens, participe à une évaporation de l'histoire (Barthes, 1957) et permet aussi de dissimuler les luttes sociales engendrées par les processus de gentrification. Il relève aussi de la «nostalgie imaginée », une nostalgie sans mémoire ni expérience vécue (Appaduraï, 2001). Mais n'est-ce pas là finalement le propre de Paris, une ville dont les habitants viennent pour leur grande majorité d'ailleurs, de province, de l'étranger ou de banlieue, «le Parisien " étant ainsi quelque part toujours amené à inventer sa parisianité en puisant dans l'imaginaire très puissant que la capitale ne cesse de charrier, fonctionnant comme opérateur d'une identité à la fois lâche et forte ? Si le vécu métropolitain de ces acteurs est exclusif, il entre en résonance avec d'autres territoires métropolitains où il est possible de retrouver les mêmes modalités de l'expérience ici décrite : Lastarria-Bellas 
Artes à Santiago du Chili, Saint-Georges à Lyon ou Côte-des-Neiges à Montréal, ces quartiers en proie à des processus de gentrification offrent des espaces « à la mode » et de consommation, des espaces aussi construits pour venir éprouver l'identité d'une ville.

Au regard de la production de ce monde urbain, l'identité de « métropolitain » promue par ParisMétropole peut-elle constituer une référence pour ces Parisiens ${ }^{12}$ ? Se pose ici clairement la question de la place de Paris au sein du projet : quelle place pourait occuper Paris dans ce territoire en construction ? Paris-Métropole se construira-t-il en opposition ou sans ce monde urbain parisien? Peut-être que l'avenir du projet réside alors moins dans la problématique de l'identité des territoires déjà présente ${ }^{13}$, que dans celle de l'altérité - de la relation à l'Autre et aux autres -, autrement dit du sens social donné au projet.

\section{$R \cdot E \cdot F \cdot E \cdot R \cdot E \cdot N \cdot C \cdot E \cdot S$}

APPADURAI, A. 2001. Après le colonialisme. Les conséquences culturelles de la globalisation, Paris, Ed. Payot.

BARTHES, R.. 1957. Mythologies, Paris, Éd. du Seuil, Coll. Points Essais.

BENJAMIN, W. 1991. Écrits français, trad. franç., Paris, Gallimard, coll. « Bibliothèques des idées $»$.

BOURDIN, A. 2005. La métropole des individus, Paris, Ed. de l'Aube.

CHALVON-DEMERSAY, S. 1984. Le triangle du XVI . Des nouveaux habitants dans un vieux quartier de Paris, Paris, Éd. de la MSH.

CORBILLÉ, S. 2009. « Tourisme, diversité enchantée et rapports symboliques dans les quartiers gentrifiés du nord-est de Paris », Genèses, 76, pp. 30-51.

DONZELOT, J. 2004, « La ville à trois vitesses : gentrification, relégation, périurbanisation », Esprit, 303, pp. 14-39.

HERPIN, N. 1986. « Socio-style », Revue française de sociologie, 27, pp. 265-272.

LAHIRE, B. 2004. La culture des individus. Dissonances culturelles et distinction de soi, Paris, La Découverte.

LALLEMENT, E. 2009. La ville marchande. Enquête à Barbès, Paris, Téraèdre.

MICOUD, A. 1991. «Les lieux exemplaires : des lieux pour faire croire à de nouveaux espaces "), in André MICOUD, (dir.), Des Hauts-Lieux. La construction sociale de l'Exemplarité, Paris, Ed. du CNRS, pp. 53-63.

MONGIN, O.. 2004. « La mondialisation et les métamorphoses de l'urbain. Mégavités, « villes globales » et métropoles », Esprit, 303, 
pp. 175-198.

RONCAYOLO, M. 1994. «Une géographie symbolique en devenir », Le Débat, 80, p. 318.

SENNETT, R. 1979 [1974]. Les tyrannies de l'intimité, Paris, Éd. du Seuil.

SIMMEL, G. 1988. « La mode », in La tragédie de la culture et autres essais, trad. franç., Paris, Rivages, pp. 88-126 ; et 2004 [1979]. « Métropole et mentalité », in Y. Grafmeyer et I. Joseph (textes traduits et présentés par), L'École de Chicago. Naissance de l'écologie urbaine, Paris, Flammarion, pp. 61-77.
$\mathrm{N} \cdot \mathrm{O} \cdot \mathrm{T} \cdot \mathrm{E} \cdot \mathrm{S}$

1. Voir l'étude «Une histoire croisée de Paris et de ses banlieues à l'époque contemporaine. Bilan d'étape », réalisée pour la ville de Paris par Annie Fourcaut et Mathieu Flonneau, Centre d'histoire sociale du XX $\mathrm{X}^{\mathrm{e}}$ siècle université Paris 1 Panthéon/ Sorbonne, septembre 2005. 2. Ce sont là des expressions que l'on retrouve fréquemment dans la bouche des acteurs de la Ville de Paris, par exemple à l'occasion des rencontres « Entre Villes et métropoles » organisées par la Ville de Paris et la Maison de l'Europe.

3. L'enquête ethnographique s'est déroulée entre 2001 et 2006 et a essentiellement porté sur les $\mathrm{X}^{\mathrm{e}}, \mathrm{XI}^{\mathrm{e}}, \mathrm{XII}^{\mathrm{e}}$, $\mathrm{XX}^{\mathrm{e}}$ et $\mathrm{XIX}^{\mathrm{e}}$ arrondissements Elle a reposé sur l'observation de situations résidentielles, marchandes ou encore touristiques et sur la réalisation d'une cinquantaine d'entretiens avec divers acteurs (habitants, commerçants, politiques, etc.). Cette première enquête a donné lieu à d'autres recherches, notamment dans le cadre de contrats de recherche avec la Ville de Paris et le ministère de la Culture portant sur les commerces et la ville. 4. Sur l'histoire de cette opposition, voir M. Agulhon, « Paris. La traversée d'est en ouest », in 1992. P. Nora (dir.). Les Lieux de mémoire, III, Les France, vol. 3, pp. 871-872.

5. Je me réfère à la définition de Jean Bazin : "L'ensemble de ce qui pour des acteurs quelconques va de soi a la forme d'un monde: ils sont dedans, ils y vivent. C'est l'ensemble de ce qu'ils présupposent et répètent par prétérition chaque fois qu'ils agissent, de ce sur quoi ils s'entendent, y compris dans leurs conflits, sans en avoir jamais convenu. ", "Science des mœurs et description de l'action », in Le genre humain, Paris, Éd. du Seuil, hiver 1999-printemps 2000, p. 42.

6. Voir F. Dubet : "Si la notion d'expérience peut paraître plus adéquate que celle de rôle et de statut, 
c'est, dans une large mesure, parce qu'elle évoque une hétérogénéité $d u$ "vécu», une diversité, là où la notion de rôle suggère d'abord la cohérence et l'ordre (...)», in 1995. Sociologie de l'expérience, Paris, Seuil, « La couleur des idées », p. 174.

7. Chalvon-Demersay, S. 1984. Le triangle $d u X V I^{e}$. Des nouveaux habitants dans un vieux quartier de Paris, Paris, Éd. de la MSH, pp.31-38

8. Voir G. Simmel, 2004. Et aussi F. Asher : « De vrais voisins métropolitains sont des voisins qui s'ignorent », in 1995. Métapolis ou l'avenir des villes, Paris, Odile Jacob, p. 16. Cela n'est pas confirmé par l'enquête, bien au contraire.

9. J'emprunte cette métaphore à B. Lahire qui la mobilise pour décrire la spécificité de l'individu contemporain dont les différentes dimensions de sa vie sociale et personnelle sont repliées en lui, qui a intériorisé la pluralité des logiques sociales, in 1998. L'homme pluriel. Les ressorts de l'action, Paris, Nathan.

10. Emmanuelle WaLter, « Les pionniers de l'Est ont la pêche », TéléObs Cinéma, dossier « De Tolbiac à Marne-la-Vallée, de Vincennes à Nogent. . . la ruée vers l'est », 1878, du 2 au 8 novembre 2000, pp. II-III.

11. Le document le Plan programme de l'est de Paris visant à rééquilibrer la capitale au début des années 80 , est révélateur de la valorisation de ces quartiers qui passe par une reconnaissance de leur histoire et de leur pleine appartenance à Paris. Le document identifie ainsi des sites « méconnu[s] et mal exploité[s] » parmi lesquels figurent « la Seine dont il n'est jusqu'à présent tiré aucun parti dans ce secteur de la ville», «les canaux et les bassins (Saint-Martin; La Villette; Ourcq ; Saint-Denis) » et «les collines de Belleville ou de Ménilmontant dont le relief a été malheureusement nié ou mal pris en compte ». Mais aussi «une série de sites ou d'espaces moins vastes, mais également très remarquables du fait de leur caractère pittoresque, leur signification historique, la valeur de leurs monu- ments » par exemple le parc des Buttes Chaumont, le cimetière du Père Lachaise ou les places de la Bastille, de la Nation ou de la République. «Enfin, malgré la dégradation complète de certaines parties du tissu urbain, (...) malgrél'état disparate qui en résulte pour ces quartiers et l'impression de moindre attention parfois de semi-abandon-qu'ils peuvent donner, l'est de Paris représente aussi un élément d'originalité, de vie, d'animation qui participe à l'image d'ensemble de la capitale. Il continue de comprendre tout un réseau d'anciens villages, faubourgs, lotissements, tout un tracé de voies traditionnelles, intéressants non pour la qualité architecturale de tel ou tel bâtiment, mais par leur caractère d'ensemble. L'intérêt de ses éléments spécifiques semble d'autant plus élevé que leur importance n'est aujourd'hui plus très considérable, alors même qu'ils demeurent essentiels pour donner à l'est de Paris son poids d'histoire, sa bonhomie, son côté attachant et sa valeur architecturale, urbaine et humaine. », in Paris Projet, Paris, APUR, 27-28, 1987, pp.10-18. Les sept arrondissements concernés sont les $\mathrm{X}^{\mathrm{e}}, \mathrm{XI}^{\mathrm{e}}, \mathrm{XII}^{\mathrm{e}}, \mathrm{XIII}{ }^{\mathrm{e}}, \mathrm{XVIII}^{\mathrm{e}}, \mathrm{XIX}^{\mathrm{e}}, \mathrm{XX}^{\mathrm{e}}$.

12. Cela est vrai de ceux qui vivent à Paris mais aussi de ceux qui vivent en banlieue. À cet égard, les films mis en ligne sur le site Internet de la ville de Paris visant à donner la parole aux habitants de la métropole sont révélateurs : le Paris intra-muros est dans la plupart des cas présenté comme différent, à part, voire exceptionnel.

13. Sur ce point, l'analyse de Marc Augé reste tout à fait éclairante. Selon lui, le durcissement observé des catégories témoigne de "l'emprise de la logique de l'identité sur celle de l'altérité : faute de pouvoir penser l'autre comme autre (celui qui n'est ni semblable à moi, ni différent de moi, et qui est donc lié à moi), on en fait un étranger ", in 1994. Pour une anthropologie des mondes contemporains, Paris, Champs Flammarion, pp. 88-89. 
$R \cdot E ́ \cdot S \cdot U \cdot M \cdot E$

À partir d'une enquête ethnographique menée dans les quartiers du nord-est de Paris, cet article analyse le vécu métropolitain des habitants appartenant aux nouvelles classes moyennes et supérieures venus s'installer depuis plusieurs décennies autour des rues de faubourg et des anciens villages. En premier lieu, leur vécu métropolitain repose sur une forme de vie urbaine dans laquelle on trouve à la fois l'expression d'un mode de vie, l'instauration d'un ordre social urbain et l'attachement au « local», mais aussi le fait de vivre une « expérience ». En second lieu, dans la mesure où vivre dans ce monde urbain, c'est aussi affirmer pleinement son identité de Parisien au sens de faire l'expérience à la fois d'une ville et de « la grande ville », leur métropole s'arrête souvent aux frontières de Paris. Dès lors, ce monde urbain semble résister au projet de Paris-Métropole de créer de l'appartenance commune.

\section{Summary}

From an ethnographic study conducted in the northeast districts of Paris, this article aims to analyze the experiences of metropolitan inhabitants belonging to the new middle and upper classes who have settled in the faubourg streets and former villages of the city over the past several decades. The lived metropolitan experience of these individuals is primarily based on a form of urban life in which there is an expression of lifestyle, the installation of an urban social order and the commitment to a "local" ideal, while focusing on living an "experience". To live in this urban environment it implies also fully asserting one's identity as a Parisian as a means to experience a specific city and a large metropolitan area. Their city often stops at the borders of Paris. Therefore, this urban environment seems to resist the Paris-Métropole project so as to create a sense of community. 